\title{
Spherical Fast Multiscale Approximation by Locally Compact Orthogonal Wavelets
}

\author{
by \\ Frank Bauer $^{1)}$ and Martin Gutting ${ }^{2)}$ \\ 1) Fuzzy Logic Laboratorium Linz-Hagenberg \\ University of Linz \\ Softwarepark 21 \\ 4232 Hagenberg \\ Austria \\ Email: frank.bauer@jku.at \\ 2) Geomathematics Group \\ University of Kaiserslautern \\ P.O. Box 3049 \\ 67653 Kaiserslautern \\ Germany \\ Email: gutting@mathematik.uni-kl.de
}

\begin{abstract}
Using a stereographical projection to the plane we construct an $\mathcal{O}(N \log (N))$ algorithm to approximate scattered data in $N$ points by orthogonal, compactly supported wavelets on the surface of a 2 -sphere or a local subset of it. In fact, the sphere is not treated all at once, but is split into subdomains whose results are combined afterwards. After choosing the center of the area of interest the scattered data points are mapped from the sphere to the tangential plane through that point. By combining a $k$-nearest neighbor search algorithm and the two dimensional fast wavelet transform a fast approximation of the data is computed and mapped back to the sphere.

The algorithm is tested with nearly 1 million data points and yields an approximation with $0.35 \%$ relative errors in roughly 2 minutes on a standard computer using our MATLABRimplementation. The method is very flexible and allows the application of the full range of two dimensional wavelets.
\end{abstract}

Keywords Orthogonal Wavelets on the Sphere, Spherical Multiresolution Analysis, Fast Wavelet Transform, Scattered Data, Approximation of Spherical Functions.

Subject classification $42 \mathrm{C} 40,43 \mathrm{~A} 90,65 \mathrm{D} 10,86-08,65 \mathrm{~T} 60$

Final paper available at http://www.springerlink.com 


\section{Introduction}

In many modern applications in geosciences one requires good approximation procedures on the sphere for large amounts of data. Whereas global methods like approximation with spherical harmonics have proven to be reliable and fast for global prediction $[22,23]$ the focus for regional reconstructions has shifted to more localizing techniques like wavelets or splines $[11,25,27]$.

The wavelet approximation on $\mathbb{R}^{2}$ is very well studied (see e.g. [19]), there exists a huge number of wavelets for very different purposes. In particular there are a number of wavelets which are orthogonal or fulfill other very interesting properties such as compact support (cf. [10]). Specifically the usage of filter banks makes a 2D wavelet transform very fast. Another particular advantage is that one can deal with data which are not given on a specific grid, e.g. by lifting schemes [16], the speed disadvantage in comparison to grid based methods is manageable. Another possibility is using non-grid based FFT techniques [21] and going to the wavelet transform via the known representation in the Fourier domain.

The situation on the sphere is rather different. All wavelet methods there suffer from one specific property of the sphere, it is not parallelizable. Therefore, there cannot be any coordinate based wavelet transform covering the whole sphere. Usually one feature of wavelets is that a single function, the so-called mother-wavelet (see e.g. [10]) is dilated to cover different frequency bands and is shifted to cover the spatial domain. Any locally supported function on the sphere with small support cannot be shifted around on the sphere in order to cover it without overlap. This limits considerably the usage of a mother-wavelet because one has to solve rather big systems of equations to counter the hereby introduced non-orthogonality of the wavelets if one uses a classically inspired approach.

Due to the complexity of introducing wavelets on the sphere a number of different approaches have been developed. Orthogonality has been shown between the different scales of an isotropic wavelet which has the standard translation and scaling properties [11]. By accepting non-isotropy of the wavelet more orthogonality can be achieved (see [12]). One particular advantage of all these wavelets is that they are physically meaningful. Another approach is the use of the Dunkl setting to achieve invariance under finite reflection groups (cf. [6] and the references therein) which finds applications in crystallography.

In a series of articles $[1,2,3]$ abstract conditions for wavelets on manifolds are developed and corresponding wavelets are constructed. However, these lack of certain deficiencies which limit their applicability in practice. Namely one would require integrations on the sphere, which is due to unknown integration weights (unless one is on very special grids) highly cost intensive. Furthermore, it is not immediately clear how one can make these physically meaningful, i.e. allow for an easy harmonic continuation to the 3 dimensional space.

Other approaches are based on a spherical harmonics expansion [20, 27, 28]. Such an approach poses rather big problems when one wants to have compact support and again suffers from the need of fast algorithms for the wavelet transform. Others have created wavelets based on tensor products [9] or operate on subdomains [8, 15] or use other analytical construction methods [13]. The vast field of boundary integral equations 
has also led to the creation of wavelet methods and fast wavelet algorithms on manifolds (see e.g. [14, 17]).

What remains is that there is no possibility to construct isotropic wavelets on the sphere which are orthogonal. So we will drop isotropy on the sphere in favor for some transferred isotropy from $\mathbb{R}^{2}$ and orthogonality. We will provide a wavelet transform on the plane which infers all important properties to the sphere including the ability for easy harmonic extension. Due to its base on the plane integration and wavelet transforms can be done very fast and efficiently without relying on specific integration grids on the sphere. Obviously, we are focussed on applications that have to deal with large amounts of spherical data, e.g. as part of the treatment of satellite data (that has been corrected for its temporal variations). However, since our method is based on locally compact wavelets it is also possible to treat domains that cover only a part of the sphere. Because of the great speed of the algorithm it is very suited as a tool to quickly get a first approximation, thereby sacrificing some accuracy that might be gained by using other methods with a higher computational effort.

In Section 2 we will shortly discuss the properties of the Kelvin transform between sphere and plane, in the next one (Section 3) we will give a construction how to convert $2 \mathrm{D}$-wavelets to spherical wavelets. Before we provide the proofs of selected lemmas (Section 6) we will show an example application of approximating the so-called disturbing potential of the Earth's gravitational field from large sets of scattered data points (Section 4).

\section{Mapping the Sphere to the Plane and Back}

Let $\Omega$ be the 2 -sphere embedded in $\mathbb{R}^{3}$ with center 0 and radius 1 . Points on $\Omega$ can be described by the parametrization with latitude $0 \leq \varphi \leq \pi$ and longitude $0 \leq \psi \leq 2 \pi$ such that their coordinates in $\mathbb{R}^{3}$ are provided by $\eta=\eta(\varphi, \psi)=(\sin \varphi \cos \psi, \sin \varphi \sin \psi, \cos \varphi)^{T}$. As usual, the space of all square-integrable functions is denoted by $L^{2}(\Omega)$ using the canonical inner product

$$
\langle f, g\rangle_{L^{2}(\Omega)}=\int_{\Omega} f(\eta) g(\eta) d \omega(\eta) .
$$

A well-known complete orthonormal basis system for $L^{2}(\Omega)$ are the spherical harmonics $\left\{Y_{n, k}\right\}_{n \in \mathbb{N},|k| \leq n}$. The spherical harmonics $Y_{n, k}$ of degree $n \in \mathbb{N}$ can be defined as the $2 n+1$ orthonormal eigenfunctions of the Beltrami operator $\Delta^{*}$ to the eigenvalue $-n(n+1)$ where $\Delta^{*}$ is the angular part of the Laplace operator. It should be pointed out that spherical harmonics are polynomials and therefore, the whole sphere is their support. Obviously, spherical harmonics can be used for Fourier analysis on the sphere.

Right now we will introduce the map we will use for later constructions. We cannot (sensibly) map the whole sphere to $\mathbb{R}^{2}$ but we need to define an area of interest which can easily extend to more than half of the sphere.

Definition 2.1 (Inversion wrt. a Sphere) The point $\gamma \in \Omega \subset \mathbb{R}^{3}$ should denote the center of the area of interest. Then define the inversion on the sphere

$$
\Upsilon_{\gamma}: \mathbb{R}^{3} \backslash\{-\gamma\} \rightarrow \mathbb{R}^{3} \backslash\{-\gamma\}
$$


by

$$
\Upsilon_{\gamma}: \chi \mapsto \Upsilon_{\gamma}(\chi)=4 \frac{\chi+\gamma}{|\chi+\gamma|^{2}}-\gamma
$$

While $\Upsilon_{\gamma}$ is defined on almost all of $\mathbb{R}^{3}$, we are interested only in the restriction $\Upsilon_{\gamma}$ : $\Omega \backslash\{-\gamma\} \rightarrow P_{\gamma}$, where $P_{\gamma}$ denotes the tangential plane to the sphere $\Omega$ through the point $\gamma$.

Remark 2.2 The inversion wrt. a sphere maps spheres to spheres (a plane is a sphere with infinite radius) [7]. Therefore it also maps circles to circles (where again a line is a circle with infinite radius).

This special inversion wrt. a sphere is also known under the name stereographical projection.

Definition 2.3 (Kelvin Transform) The point $\gamma \in \Omega$ should denote the center of the area of interest. Let $\varepsilon>0$ and $B_{\varepsilon}(-\gamma)$ denotes the ball of radius $\varepsilon$ around $-\gamma$. The Kelvin transform of the function $v \in L^{2}\left(\Omega \backslash B_{\varepsilon}(-\gamma)\right)$ is defined by

$$
\Theta_{\gamma}[\cdot]: L^{2}\left(\Omega \backslash B_{\varepsilon}(-\gamma)\right) \rightarrow L^{2}\left(P_{\gamma}\right), \quad \Theta_{\gamma}[v](\cdot)=\frac{2}{|\cdot+\gamma|} v\left(\Upsilon_{\gamma}(\cdot)\right)
$$

The inversion wrt. a sphere with center $-\gamma$ as defined above and the corresponding Kelvin transform fulfill the following properties, the proofs of the subsequent can be found in Section 6.

Lemma 2.4 (Properties) The following properties hold:

$$
\begin{aligned}
& \Upsilon_{\gamma}\left(\Upsilon_{\gamma}(\chi)\right)=\chi \\
& \Theta_{\gamma}\left[\Theta_{\gamma}[v]\right]=v
\end{aligned}
$$

The map $\Theta_{\gamma}$ is linear, i.e. for any constant $c \in \mathbb{R}$

$$
\Theta_{\gamma}[c v+u]=c \Theta_{\gamma}[v]+\Theta_{\gamma}[u]
$$

Furthermore $\gamma$ is a fixed point and formally $-\gamma$ is mapped to $\infty$.

Remark 2.5 Additionally, the Kelvin transform maps harmonic functions to harmonic functions [4].

Lemma 2.6 (Functions) Let $\Gamma \subset \Omega \backslash B_{\varepsilon}(-\gamma)$ with an $\varepsilon>0$ by any measurable subset. Assume that we use a longitude-latitude parameterization of the sphere $\Omega$ with latitude $0 \leq \varphi \leq \pi$ and longitude $0 \leq \psi \leq 2 \pi$ and a standard $(x, y)$ cartesian coordinate system of the plane we map $\Gamma$ to.

Then it holds:

$$
\begin{aligned}
& \Upsilon_{\gamma}((\varphi, \psi))=\left(2 \tan \frac{\varphi}{2} \cos \psi, 2 \tan \frac{\varphi}{2} \sin \psi\right) \\
& \Upsilon_{\gamma}((x, y))=\left(2 \arctan \frac{\sqrt{x^{2}+y^{2}}}{2}, \arctan \frac{y}{x}\right)
\end{aligned}
$$




$$
\begin{aligned}
& \Theta_{\gamma}[f]((\varphi, \psi))=\frac{\sqrt{2}}{\sqrt{1+\cos \varphi}} f\left(\Upsilon_{\gamma}((\varphi, \psi))\right) \\
& \Theta_{\gamma}[f]((x, y))=\frac{2}{\sqrt{r^{2}+4}} f\left(\Upsilon_{\gamma}((x, y))\right)
\end{aligned}
$$

using $r=\sqrt{x^{2}+y^{2}}$.

Lemma 2.7 (Integrals) For any measurable subset $\Gamma \subset \Omega \backslash B_{\varepsilon}(-\gamma)$ with an $\varepsilon>0$

$$
\int_{\Gamma} f(\xi) d \omega(\xi)=\int_{(\varphi, \psi) \in \Gamma} f(\varphi, \psi) \sin \varphi d \varphi d \psi=\int_{(x, y) \in \Upsilon_{\gamma}(\Gamma)} f\left(\Upsilon_{\gamma}(x, y)\right) w(x, y) d x d y
$$

where

$$
w(x, y)=\left(\frac{4}{x^{2}+y^{2}+4}\right)^{2}=\left(\frac{4}{r^{2}+4}\right)^{2} .
$$

Furthermore it holds for the inner products

$$
\int_{\Gamma} f(\xi) g(\xi) d \omega(\xi)=\int_{(x, y) \in \Upsilon_{\gamma}(\Gamma)} \Theta_{\gamma}[f](x, y) \Theta_{\gamma}[g](x, y) W(x, y) d x d y
$$

where

$$
W(x, y)=\frac{4}{x^{2}+y^{2}+4}=\frac{4}{r^{2}+4} .
$$

Lemma 2.8 (Derivatives) For the derivatives it holds

$$
\begin{aligned}
\frac{\partial}{\partial \varphi} f(\varphi, \psi)= & \frac{1}{\sqrt{2}} \frac{\sin \varphi}{(1+\cos \varphi)^{3 / 2}} \Theta_{\gamma}[f]\left(\Upsilon_{\gamma}(\varphi, \psi)\right) \\
& +\frac{\sqrt{2} \cos \psi}{\sqrt{1+\cos \varphi} \cos ^{2} \frac{\varphi}{2}} \frac{\partial}{\partial x} \Theta_{\gamma}[f]\left(\Upsilon_{\gamma}(\varphi, \psi)\right) \\
& +\frac{\sqrt{2} \sin \psi}{\sqrt{1+\cos \varphi} \cos ^{2} \frac{\varphi}{2}} \frac{\partial}{\partial y} \Theta_{\gamma}[f]\left(\Upsilon_{\gamma}(\varphi, \psi)\right), \\
\frac{\partial}{\partial \psi} f(\varphi, \psi)= & -\frac{2 \sqrt{2} \tan \frac{\varphi}{2} \sin \psi}{\sqrt{1+\cos \varphi}} \frac{\partial}{\partial x} \Theta_{\gamma}[f]\left(\Upsilon_{\gamma}(\varphi, \psi)\right) \\
& +\frac{2 \sqrt{2} \tan \frac{\varphi}{2} \cos \psi}{\sqrt{1+\cos \varphi}} \frac{\partial}{\partial y} \Theta_{\gamma}[f]\left(\Upsilon_{\gamma}(\varphi, \psi)\right) .
\end{aligned}
$$

Remark 2.9 The vector fields generated by the surface curl operator $L_{\xi}^{*}$ and surface gradient operator $\nabla_{\xi}^{*}$ on the sphere follow circles or are tangential to circles (see [11] for further details). Therefore, they map to vector fields in the plane which follow circles respectively are tangential. 


\section{Wavelets}

As the literature concerning wavelets on $\mathbb{R}^{2}$ is manifold (see e.g. $[10,19]$ ) we just want to state the following:

Lemma 3.1 There exist biorthogonal families of wavelets $\Psi_{j}$ and scaling functions $\Phi_{0}$, i.e. $\left\{\left\{\Psi_{j}[n, m]\right\},\left\{\Phi_{0}[n, m]\right\}\right\}$, with compact support on $\mathbb{R}^{2}$ which allow a fast wavelet transform of a function $F \in L^{2}\left(\mathbb{R}^{2}\right)$ with

$$
F=\sum_{n, m} a_{0}[n, m] \Phi_{0}[n, m]+\sum_{j} \sum_{n, m} a_{j}[n, m] \Psi_{j}[n, m]
$$

where

$$
\begin{aligned}
& a_{j}[n, m]=\left\langle F, \Psi_{j}[n, m]\right\rangle_{L^{2}\left(\mathbb{R}^{2}\right)} \\
& a_{0}[n, m]=\left\langle F, \Phi_{0}[n, m]\right\rangle_{L^{2}\left(\mathbb{R}^{2}\right)} .
\end{aligned}
$$

In order to generate wavelets on the sphere we need to pull them back via the Kelvin transform, i.e.

Definition 3.2 Let $\left\{\left\{\Psi_{j}[n, m]\right\},\left\{\Phi_{0}[n, m]\right\}\right\}$ a family of wavelets defined on $P_{\gamma}$. Then define

$$
\widetilde{\Psi_{j}[n, m]}=\Theta_{\gamma}\left[\Psi_{j}[n, m] W^{-1 / 2}\right]
$$

respectively

$$
\widetilde{\Phi_{0}[n, m]}=\Theta_{\gamma}\left[\Phi_{0}[n, m] W^{-1 / 2}\right]
$$

on the sphere $\Omega$

Lemma 3.3 The family $\left\{\left\{\widetilde{\Psi_{j}[n, m]}\right\},\left\{\widetilde{\Phi_{0}[n, m]}\right\}\right\}$ is orthonormal on $\Omega$ if and only if $\left\{\left\{\Psi_{j}[n, m]\right\},\left\{\Phi_{0}[n, m]\right\}\right\}$ is orthonormal on $P_{\gamma} \sim \mathbb{R}^{2}$.

Proof:

We just show one of the three equations, the other ones are essentially the same

$$
\int_{\Omega} \widetilde{\Psi_{j}[n, m]}(\xi) \widetilde{\Psi_{i}[k, l]}(\xi) d \omega(\xi)=\int_{P_{\gamma}} \Psi_{j}[n, m](x, y) \Psi_{i}[k, l](x, y) d x d y=\delta_{i, j} \delta_{n, k} \delta_{m, l}
$$

The first equality holds because of (14), (12), (3) and (4)

Lemma 3.4 The wavelet transform with respect to the family $\left\{\left\{\widetilde{\Psi_{j}[n, m]}\right\},\left\{\widetilde{\Phi_{0}[n, m]}\right\}\right\}$ of the function $f$ is equivalent to the wavelet transform with respect to the family $\left\{\left\{\Psi_{j}[n, m]\right\},\left\{\Phi_{0}[n, m]\right\}\right\}$ of the function $\Theta_{\gamma}[f] W^{1 / 2}=f\left(\Upsilon_{\gamma}\right) w^{1 / 2}$. 
Proof:

It holds

$$
\begin{aligned}
\left\langle f, \widetilde{\Psi_{j}[n, m]}\right\rangle_{L^{2}(\Omega)} & =\int_{\Omega} f(\xi) \widetilde{\Psi_{j}[n, m]}(\xi) d \omega(\xi) \\
& =\int_{P_{\gamma}} \Theta_{\gamma}[f](x, y) \Psi_{j}[n, m](x, y) W(x, y)^{1 / 2} d x d y \\
& =\left\langle\Theta_{\gamma}[f] W^{1 / 2}, \Psi_{j}[n, m]\right\rangle_{L^{2}\left(P_{\gamma}\right)}
\end{aligned}
$$

which yields the proposition.

This means in particular that one can do all the necessary computations using a fast wavelet transform [16] on the plane. No integrations on the sphere are needed.

The only theoretical drawback of this method is that the corresponding wavelets on the sphere are irregularly shaped and are specifically not fulfilling the standard relations of translation invariance and scaling invariance on the sphere. However, we still obtain a family of nested scale spaces, i.e. a multiresolution analysis (see e.g. [10]) on the punctured sphere $\Omega \backslash B_{\varepsilon}(-\gamma)$. Using a partition of unity we can combine two such multiresolution analysis on $\Omega \backslash B_{\varepsilon}(-\gamma)$ and $\Omega \backslash B_{\varepsilon}(\gamma)$, respectively. This gives us a multiresolution analysis on the whole sphere $\Omega$ and allows us to speak of a spherical multiscale approach using wavelets.

In practice two further drawbacks can occur. On the one hand the mapping from the sphere to the plane transforms regular spherical grids into rather irregular point distributions in the plane and vice versa. However, if we deal with scattered point distributions, the drawback of an irregular distribution exists right from the start. As mentioned before the whole sphere cannot be treated at once. This is easily circumvented by running wavelet approximations for domains slightly larger than half the sphere with the points of interest $\gamma$ and $-\gamma$. The two approximations are assembled afterwards.

\section{Numerical Implementation and Examples}

The algorithm for the fast spherical wavelet transformation and its application to scattered data interpolation on the sphere are presented in this section. The set of scattered points on the sphere is denoted by $X_{\Omega},\left|X_{\Omega}\right|=N$, its image in the plane is called $X_{\mathbb{R}^{2}}$, $\left|X_{\mathbb{R}^{2}}\right|=N . Y_{\mathbb{R}^{2}}$ stands for the set of points of the regular grid in the plane which is used for the wavelet transforms, $\left|Y_{\mathbb{R}^{2}}\right|=M$. The evaluation points form a regular grid on the sphere and are denoted by $Z_{\Omega},\left|Z_{\Omega}\right|=N_{\mathrm{ev}}$, their image in the plane is $Z_{\mathbb{R}^{2}},\left|Z_{\mathbb{R}^{2}}\right|=N_{\mathrm{ev}}$.

Algorithm 4.1 1. Map the points $X_{\Omega}$ and the data from the sphere to the plane using $\Upsilon_{\gamma}$ and $\Theta_{\gamma}$ (only the southern hemisphere plus some overlap, i.e. only points whose latitudes $\varphi$ fulfill $\varphi \in\left[\pi / 2-\varphi_{\text {ov }}, \pi\right]$ with the overlap parameter $\left.\varphi_{\text {ov }} \in[0, \pi / 2]\right)$.

2. Map the planar points $X_{\mathbb{R}^{2}}$ to the regular grid $Y_{\mathbb{R}^{2}}$ using the following approximation procedure:

(a) Build a 2D-tree [5] for the scattered points $X_{\mathbb{R}^{2}}$ in the plane.

(b) Search the $k$ nearest neighbors in $X_{\mathbb{R}^{2}}$ for each of the grid points in $Y_{\mathbb{R}^{2}}$ and compute the corresponding distances. 
(c) Compute the weighted mean value by

$$
F_{\text {mean }}\left(y_{j}\right)=\sum_{i=1}^{k} \frac{F\left(x_{i}\right)}{\left|y_{j}-x_{i}\right|+\alpha} / \sum_{i=1}^{k} \frac{1}{\left|y_{j}-x_{i}\right|+\alpha}
$$

for each grid point $y_{j} \in Y_{\mathbb{R}^{2}}$ with its neighbors $x_{i} \in X_{\mathbb{R}^{2}}$ and a parameter $\alpha>0$.

3. Perform the fast 2D wavelet decomposition and reconstruction for all scales with the regular grid in the plane.

4. FOR each scale DO

(a) Build a 2D-tree for the grid points $Y_{\mathbb{R}^{2}}$.

(b) Search the $k$ nearest neighbors in $Y_{\mathbb{R}^{2}}$ for each of the evaluation points in $Z_{\mathbb{R}^{2}}$ and compute the corresponding distances.

(c) Compute the weighted mean value by

$$
G_{\text {mean }}\left(z_{j}\right)=\sum_{i=1}^{k} \frac{G\left(y_{i}\right)}{\left|z_{j}-y_{i}\right|+\alpha} / \sum_{i=1}^{k} \frac{1}{\left|z_{j}-y_{i}\right|+\alpha}
$$

for each evaluation point $z_{j} \in Z_{\mathbb{R}^{2}}$ with its neighbors $y_{i} \in Y_{\mathbb{R}^{2}}$ and a parameter $\alpha>0$.

(d) Map the points $Z_{\mathbb{R}^{2}}$ and the evaluated approximation from the plane back to the sphere using $\Upsilon_{\gamma}$ and $\Theta_{\gamma}$.

5. Perform steps 1 to 4 again for the northern hemisphere and some overlap, i.e. points whose latitudes $\varphi$ fulfill $\varphi \in\left[0, \pi / 2+\varphi_{\text {ov }}\right]$.

6. Combine the northern part $G_{\text {north }}$ and the southern part $G_{\text {south }}$ by

$$
G(x)=G(\varphi, \psi)=\left\{\begin{aligned}
G_{\text {north }}(x) & \text { if } \varphi & <\frac{\pi}{2}-\varphi_{\text {ov }}, \\
w G_{\text {north }}(x)+(1-w) G_{\text {south }}(x) & \text { with } w & =\frac{\varphi-\left(\frac{\pi}{2}-\varphi_{o v}\right)}{2 \varphi_{o v}}, \\
G_{\text {south }}(x) & \text { if } \varphi & >\frac{\pi}{2}+\varphi_{\text {ov }}
\end{aligned}\right.
$$

where $\varphi \in[0, \pi]$ denotes the latitude of $x$.

Note that steps 4(a), 4(b) and even the computation of the weights in step 4(c) only have to be performed for one scale. The results can be stored and reused for all other scales.

As an example for our computations we have chosen the disturbing potential of the Earth's gravitational field as described by the EGM96 model [18], but with truncation of the spherical harmonics expansion at degree 100 (see Figure 1 which also illustrates the split into two overlapping enlarged hemispheres). The data points are randomly distributed on the sphere with uniform density function. Algorithm 4.1 allows the choice of the number of nearest neighbors $k$ which we set to 5 and the approximation parameter $\alpha$ is chosen as 0.1. The evaluation grid is a regular spherical grid with step size of 1 degree. The overlap of the two enlarged hemispheres is taken at 7.5 degrees for the 
data points and at 5 degrees for the evaluation grid. The idea of taking a slightly less overlapping evaluation domain is to avoid, i.e. to cut off, boundary effects like oscillations at the boundary of the enlarged hemispheres.
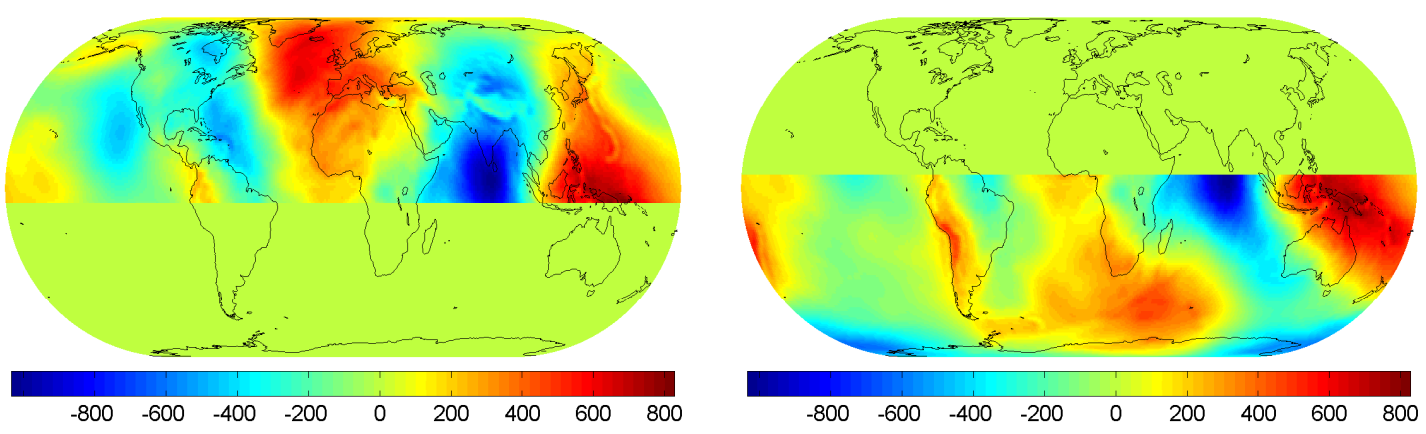

Figure 1: Original function evaluated on the two 'overlapping enlarged hemispheres', values in $\mathrm{m}^{2} / \mathrm{s}^{2}$

The following approximation results (see Figure 2 for the reconstruction, i.e. the sum of all scales, and the total absolute error) and the corresponding multiresolution (see Figure 4 and Figure 5) have been computed using 960000 scattered points on the sphere and a regular planar grid with $m=1400$ points in each dimension. The wavelet transforms are performed with symlet 4 wavelets [10].
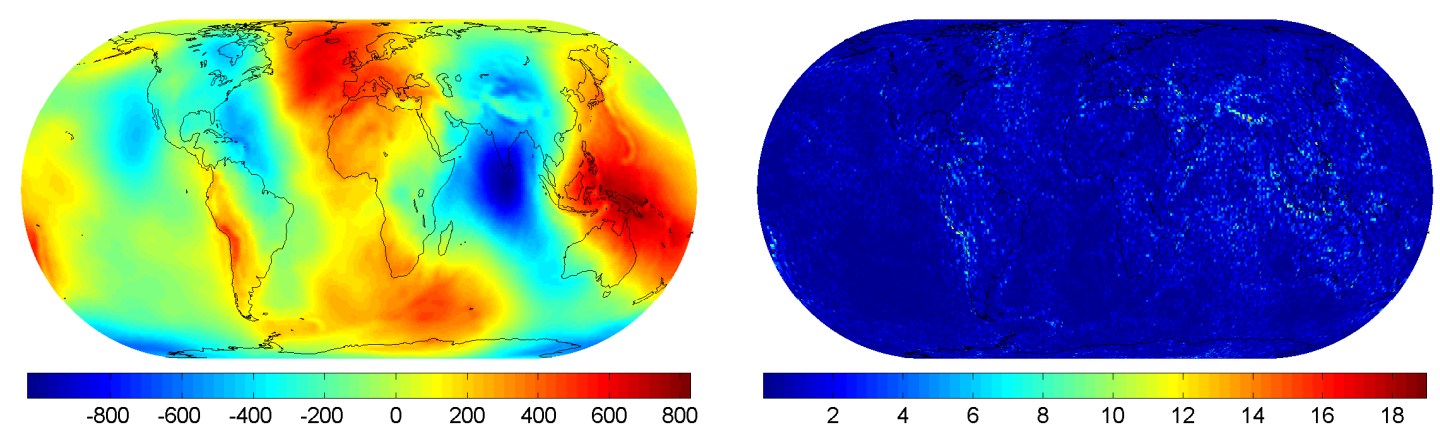

Figure 2: Reconstruction using all scales (left) and corresponding total error (right), values in $\mathrm{m}^{2} / \mathrm{s}^{2}$

In Figure 3 we separated the two sources of the total error: on the one hand the $k$ nearest neighbors approximation (left part of Figure 3) and on the other hand the wavelet decomposition and reconstruction (right part of Figure 3). Obviously, it is the approximation in the plane which causes almost all the error. The wavelet transforms work almost at machine precision (note the factor of $10^{-10}$ at the colorbar of the right image in Figure 3). However, the development of the total error is scale-dependant as it can be observed in Table 1 as well as in the multiresolution in Figure 4 and Figure 5. 

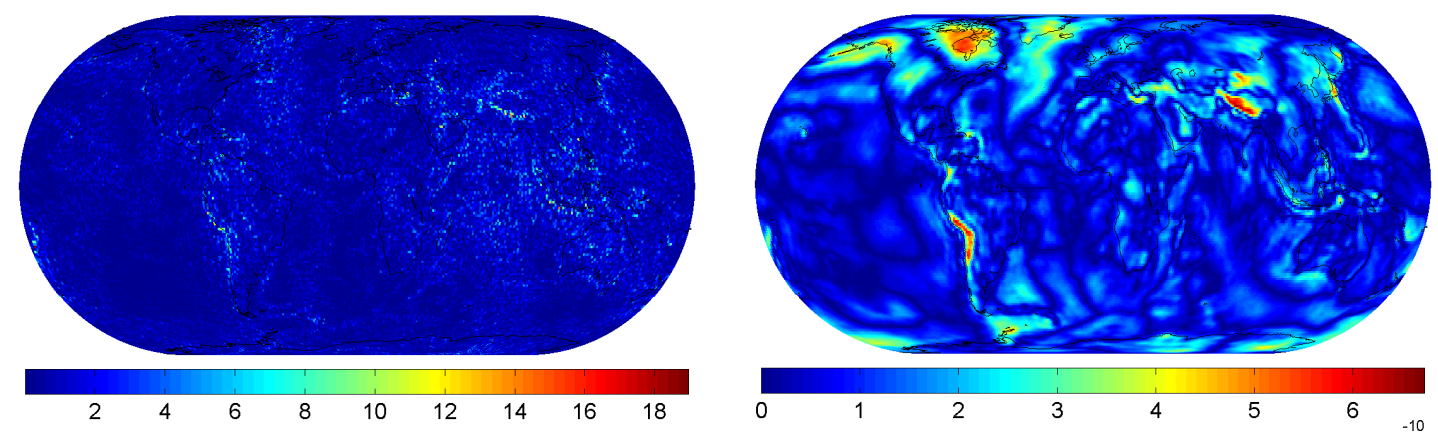

Figure 3: Approximation error without wavelet transforms (left, values in $\mathrm{m}^{2} / \mathrm{s}^{2}$ ) and error due to wavelet decomposition and reconstruction without approximation error (right, values in $10^{-10} \mathrm{~m}^{2} / \mathrm{s}^{2}$, the exponent of the factor $10^{-10}$ is indicated in the bottom right corner)

\begin{tabular}{|c|c|c|c|}
\hline Scale & mean error & rel. mean error & maximal error \\
\hline \hline 0 & 37.75 & 0.1664 & 279.35 \\
1 & 16.88 & 0.0744 & 204.53 \\
2 & 8.75 & 0.0386 & 151.01 \\
3 & 3.65 & 0.0161 & 63.825 \\
4 & 0.7516 & 0.0033 & 12.00 \\
5 & 0.525 & 0.0023 & 13.42 \\
6 & 0.724 & 0.0032 & 17.20 \\
7 & 0.8011 & 0.0035 & 18.95 \\
\hline
\end{tabular}

Table 1: Development of the mean error ( $l^{1}$-error), relative mean error (relative $l^{1}$-error) and maximal error of the approximation using only the scales up to the value in the first column. Corresponding figures are depicted in Figure 4 and Figure 5, all mean and maximal errors are in $\mathrm{m}^{2} / \mathrm{s}^{2}$.

Inherently this error which is almost completely due to the mapping of the scattered data points to a regular grid in the plane consists mainly of high frequencies which transfers to a larger error in the higher scales. Just leaving out the high-frequency scales, i.e. simple low-pass filtering, further reduces the error. A combination with a suitable wavelet denoising algorithm can probably lead to even better approximations. Such an algorithm has to be adapted to the special situation resulting from the spherical origin of the planar data. This should be investigated in the future, in particular in combination with noisy data.

Nevertheless, by taking all scales into account we obtain good results, at least for a large number of data points (see Table 2 for the development of the error for different numbers of points) and we do not have to consider specific strategies to decide how many scales should be included. 

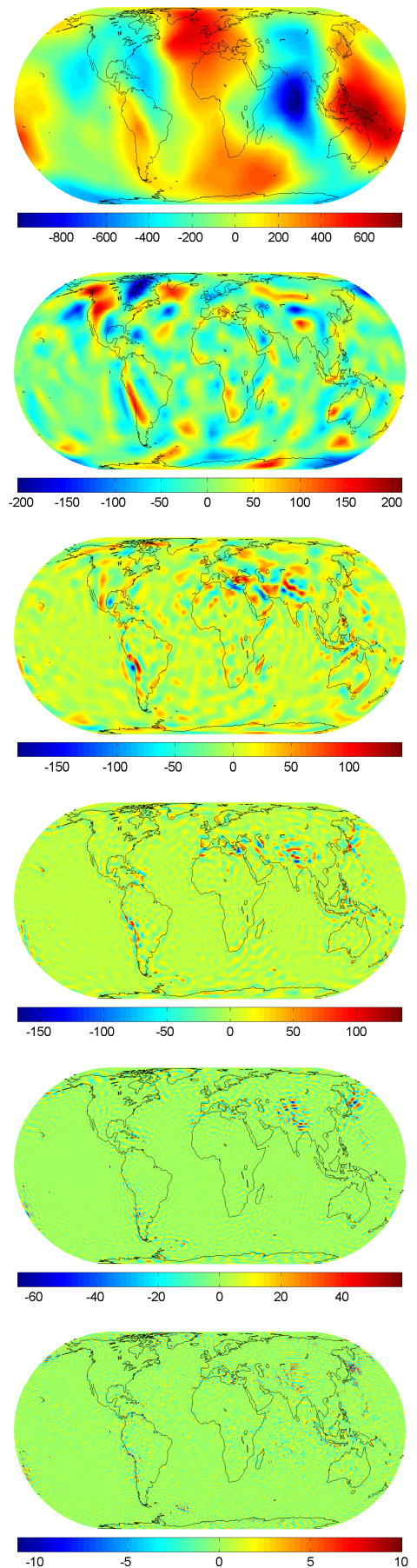
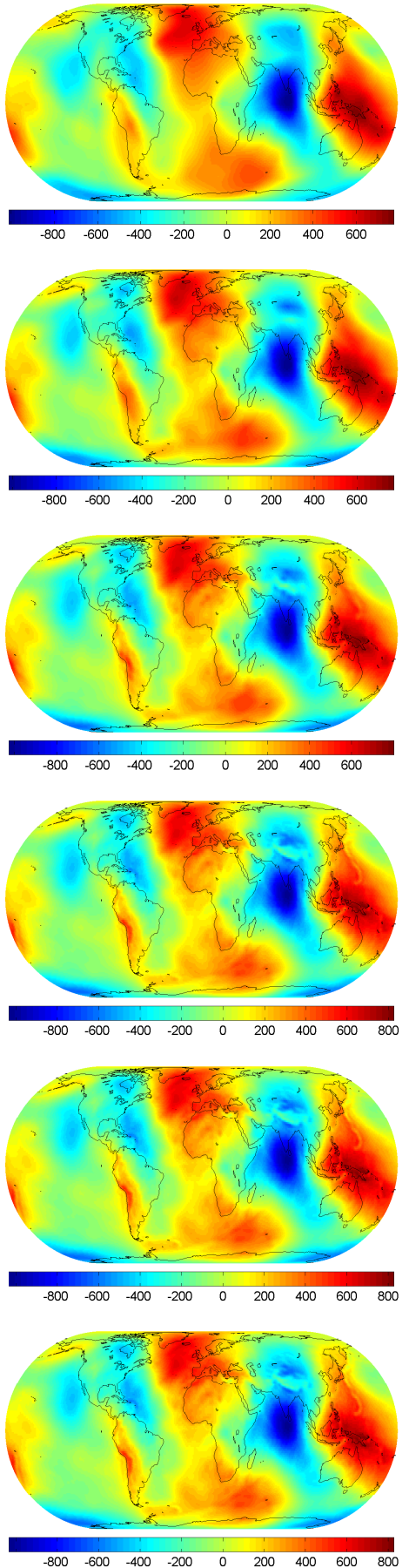
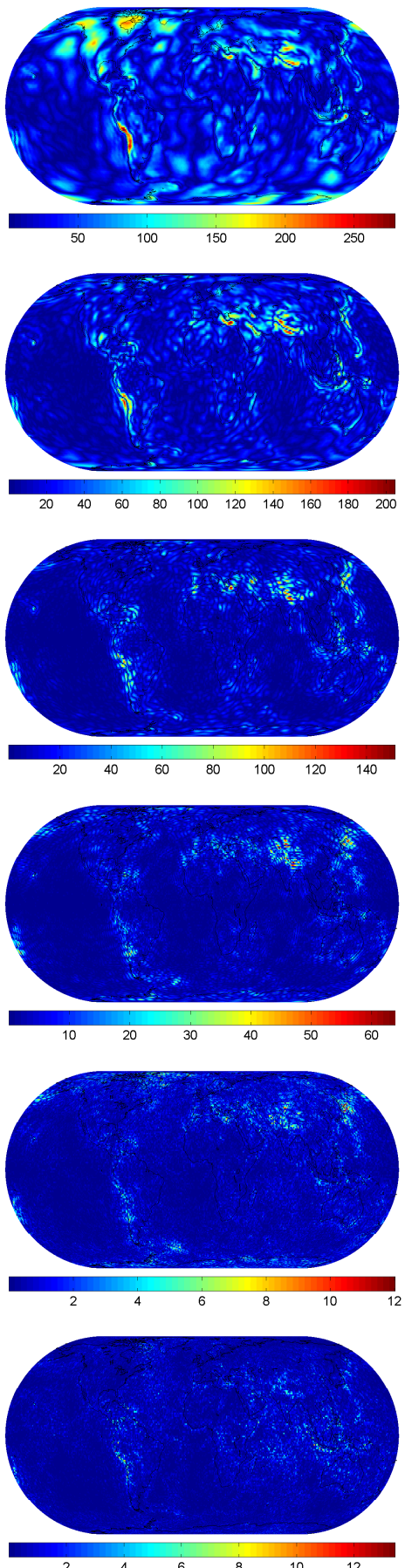

Figure 4: Multiscale decomposition into scales 0 to 5 , the left column contains the different scales in increasing order, the middle column shows the sum up to the current scale, the right column depicts the corresponding absolute error, values in $\mathrm{m}^{2} / \mathrm{s}^{2}$ 

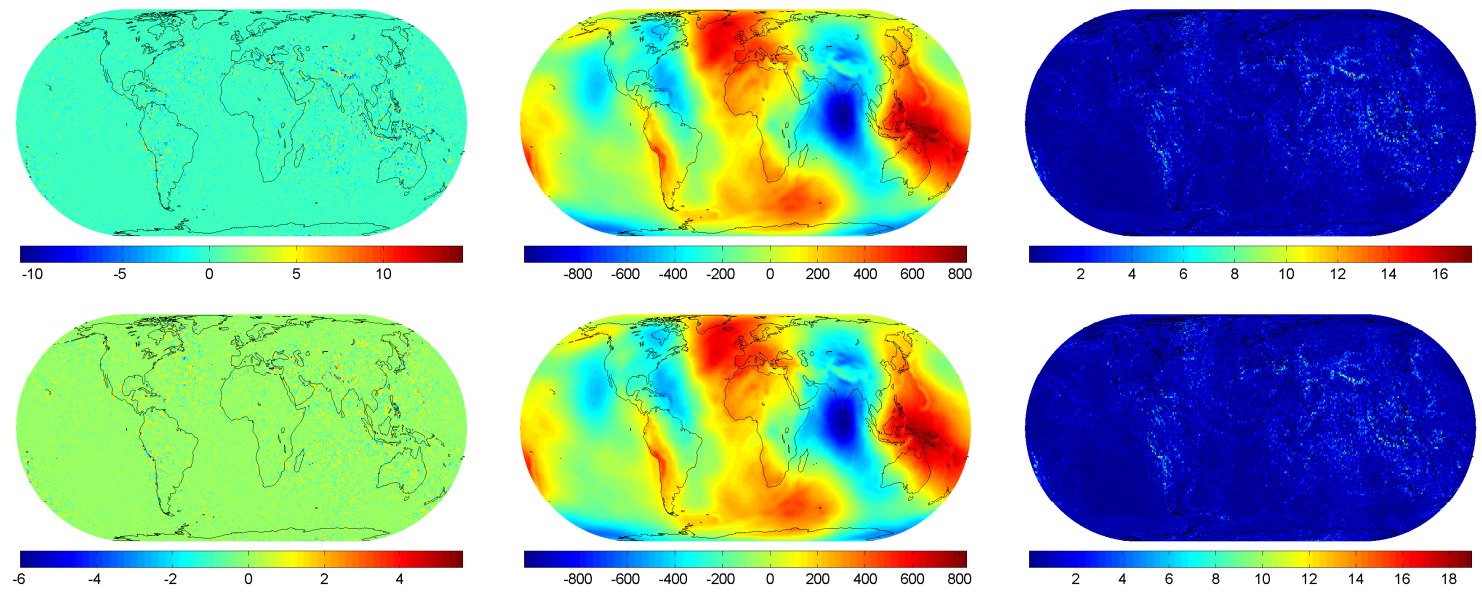

Figure 5: Multiscale decomposition into scales 6 to 7 , the left column contains the different scales in increasing order, the middle column shows the sum up to the current scale, the right column depicts the corresponding absolute error, values in $\mathrm{m}^{2} / \mathrm{s}^{2}$

Our primary intention has been to develop a fast spherical wavelet transform, i.e. an $\mathcal{O}(N \log (N))$ method. Therefore, we consider the steps of Algorithm 4.1 separately: the application of $\Upsilon_{\gamma}$ and $\Theta_{\gamma}$ in step 1 can be performed with linear effort, i.e. $\mathcal{O}(N)$ for $N$ scattered points on $\Omega$. The nearest neighbor approximation is carried out using $2 \mathrm{D}$-trees which can be built for $N$ points with $\mathcal{O}(N \log (N))$ effort in step 2(a) (see e.g. [5]). The nearest neighbor search itself (as well as the search for the $k$ nearest neighbors) for $M$ points, i.e. step 2(b), is of order $M \log (N)$ (cf. e.g. [5]) and computation of the weighted means in step $2(\mathrm{c})$ requires $\mathcal{O}(M)$ effort. During step 3 the wavelet decomposition and reconstruction is performed in $\mathcal{O}(M \log (M))$ (cf. [19]). The construction of the 2D-tree in step 4(a) is carried out with $\mathcal{O}(M \log (M))$ effort, the nearest neighbor searches for the $N_{\text {ev }}$ evaluation points in step $4(\mathrm{~b}) \operatorname{cost} \mathcal{O}\left(N_{\text {ev }} \log (M)\right)$ and the calculation of the mean values (step $4(\mathrm{c})$ ) can be performed with $\mathcal{O}\left(N_{\text {ev }}\right)$ calculations. Note that this is the only part which is required in each scale, i.e. more than once. The mapping back to the sphere in step $4(\mathrm{~d})$ is completed by $\mathcal{O}\left(N_{\text {ev }}\right)$ operations as well as the combination of the northern and the southern part in step 6 .

Altogether the main contributions are of order $\mathcal{O}(N \log (N))$ and $\mathcal{O}(M \log (M))$ since for large data sets it is safe to assume that the number of evaluation points $N_{\text {ev }}$ is less than $N$ or $M$. Therefore, the grid size has to be chosen such that $M=\mathcal{O}(N)$. Our tests have shown that a regular grid with $m \approx \sqrt{2 N}$ points in each dimension, i.e. $M \approx 2 N$, leads to good approximations without heavily affecting the performance of the algorithm.

In Table 2 we show the results of the computation of this example for different numbers of data points. The emphasis lies on the time that is consumed for the computation of the wavelet approximation on the whole sphere (column 4 of Table 2). Our computations have been performed with MATLABßon an Intel@Core 2 T7200 CPU at 2 GHz using only one of the two cores. The grid size in the plane is chosen by the aforementioned strategy and obviously the larger the grid the more scales can be calculated. The elapsed times indicate that the effort of the method is indeed of the order $N \log (N)$ with an overhead which we estimated to be 13.7 seconds. Subtracting this from the elapsed 
times the asymptotic behavior of the effort can be clearly identified (see column 5 in Table 2). For the sake of completeness the errors corresponding to different amounts of data points are also provided in the table. As before they are primarily due to the approximation procedure and obviously require a large number of scattered data points to diminish. As seen in Table 1 and the corresponding figures the errors can be further reduced by just leaving out the higher scales which are included here.

\begin{tabular}{|c|c|c|c|c|c|c|c|}
\hline \# points & grid size & scales & time & est. time & mean err. & rel. err. & max. err. \\
\hline \hline 30000 & 248 & 5 & 15.6 & 1.9 & 6.24 & 0.0275 & 103.7 \\
60000 & 350 & 5 & 18.4 & 4.7 & 4.09 & 0.0181 & 75.44 \\
120000 & 495 & 6 & 24.2 & 10.5 & 2.67 & 0.0118 & 77.18 \\
240000 & 700 & 6 & 36.7 & 23 & 1.73 & 0.0076 & 55.78 \\
480000 & 990 & 7 & 63.3 & 49.6 & 1.163 & 0.0051 & 20.26 \\
960000 & 1400 & 7 & 120.8 & 107.1 & 0.801 & 0.0035 & 18.95 \\
\hline
\end{tabular}

Table 2: Elapsed time for different numbers (column 1) of scattered points on the sphere using the corresponding grid sizes (column 2) and the corresponding number of scales (column 3). The total times are given in seconds (column 4) and without the estimated overhead time of $13.7 \mathrm{~s}$ (column 5). The other columns show the corresponding mean error ( $l^{1}$-error), relative mean (relative $l^{1}$-error) and maximal errors (columns 6 to 8 ) for the reconstruction using all scales, mean and maximal errors are in $m^{2} / s^{2}$.

\section{Conclusion and Outlook}

We have constructed a fast wavelet transform on the sphere which exhibits all properties of standard Euclidean wavelets which are relevant in practical applications, namely orthogonality and compact support. Due to the "detour" to $\mathbb{R}^{2}$ we are capable to use all fast algorithms and classes of wavelets which have been developed in Euclidean space. The preservation of harmonicity of the transform enables us (when needed) to build quickly an expansion in 3D-harmonic functions with compact support on the sphere.

The only drawback of the method is the need of using non-regular grids on the plane. However, as mostly data are given scattered and the number of good integration grids for the sphere with a high number of support points is limited (see also [29] on this matter), this also applies to all other methods. The advantage of leaving the sphere and going to the plane is the possibility to use a much richer set of methods to deal with this problem. We have applied our MATLAB@implementation to an example of scattered data interpolation and determined a multiscale approximation from nearly 1 million data points in about 2 minutes on a low-end PC with relative mean errors of $0.35 \%$. As mentioned in Section 4 the quality of the results can easily be improved further with the help of a denoising method. For now we have only considered the simplest choice of cutting off the finest details. Other denoising procedures will be investigated in the future. This also opens up the possibility to extend this method to approximation from noisy data.

Moreover, the formation of the weighted mean values in the $k$ nearest neighbors search leaves room for improvements and the corresponding parameters $k$ and $\alpha$ can surely be 
optimized. However, more nearest neighbors, i.e. a larger value for $k$, slightly increases the numerical effort.

\section{Acknowledgements}

We would like to express our gratitude to F.J. Simons [24] for publically providing a neat piece of MATLABßCode to do speedy computations with spherical harmonics and gravitation models and to A. Tagliasacchi [26] for providing a decent implementation of the KD-tree algorithm in MATLABß).

The first author was supported by the Upper Austrian Research and Technology Promotion.

\section{Proofs}

Although all proofs presented here are straightforward and not necessarily innovative we include them for the sake of completeness.

Proof of Lemma 2.4:

In order to show (3) we will restrict ourselves to the main case of (1), the two special cases are trivial.

$$
\Upsilon_{\gamma}\left(\Upsilon_{\gamma}(x)\right)=4 \frac{\left(4 \frac{x+\gamma}{|x+\gamma|^{2}}-\gamma\right)+\gamma}{\left|\left(4 \frac{x+\gamma}{|x+\gamma|^{2}}-\gamma\right)+\gamma\right|^{2}}-\gamma=4 \frac{4 \frac{x+\gamma}{|x+\gamma|^{2}}}{\left|4 \frac{x+\gamma}{|x+\gamma|^{2}}\right|^{2}}-\gamma=x+\gamma-\gamma=x .
$$

Just as well for showing (4) we will only consider the main case of (2)

$$
\Theta_{\gamma}\left[\Theta_{\gamma}[v]\right](x)=\frac{2}{|x+\gamma|} \frac{2}{\left|\left(4 \frac{x+\gamma}{|x+\gamma|^{2}}-\gamma\right)+\gamma\right|} v(x)=v(x)
$$

The next property that the sphere maps to a plane and the exterior to a half space is standard Euclidean geometry [7], the fact that harmonic functions are mapped to harmonic functions is proven in [4].

The linearity of the Kelvin transform is obvious.

Proof of Lemma 2.6:

We can easily calculate some parts (see figure 6): The radius $r=|E C|$ of the circle around $C$ is using similar triangles

$$
r(\varphi)=2 \frac{\sin \varphi}{1+\cos \varphi}=2 \sqrt{\frac{1-\cos \varphi}{1+\cos \varphi}}=2 \tan \frac{\varphi}{2}
$$

and hence (6) holds. Furthermore applying the inverse functions shows (7). The equations (8) and (9) are direct consequences of being $\gamma$ equivalent to the $z$ direction of the $(x, y)$-plane.

Proof of Lemma 2.7:

The Jacobian of (7) is for $r=\sqrt{x^{2}+y^{2}}$

$$
\left|\begin{array}{cc}
\frac{4 x}{r\left(4+r^{2}\right)} & \frac{4 y}{r\left(4+r^{2}\right)} \\
-\frac{y}{r^{2}} & \frac{x}{r^{2}}
\end{array}\right|=\frac{4 r^{2}}{r^{3}\left(4+r^{2}\right)}=\frac{4}{r\left(4+r^{2}\right)}
$$




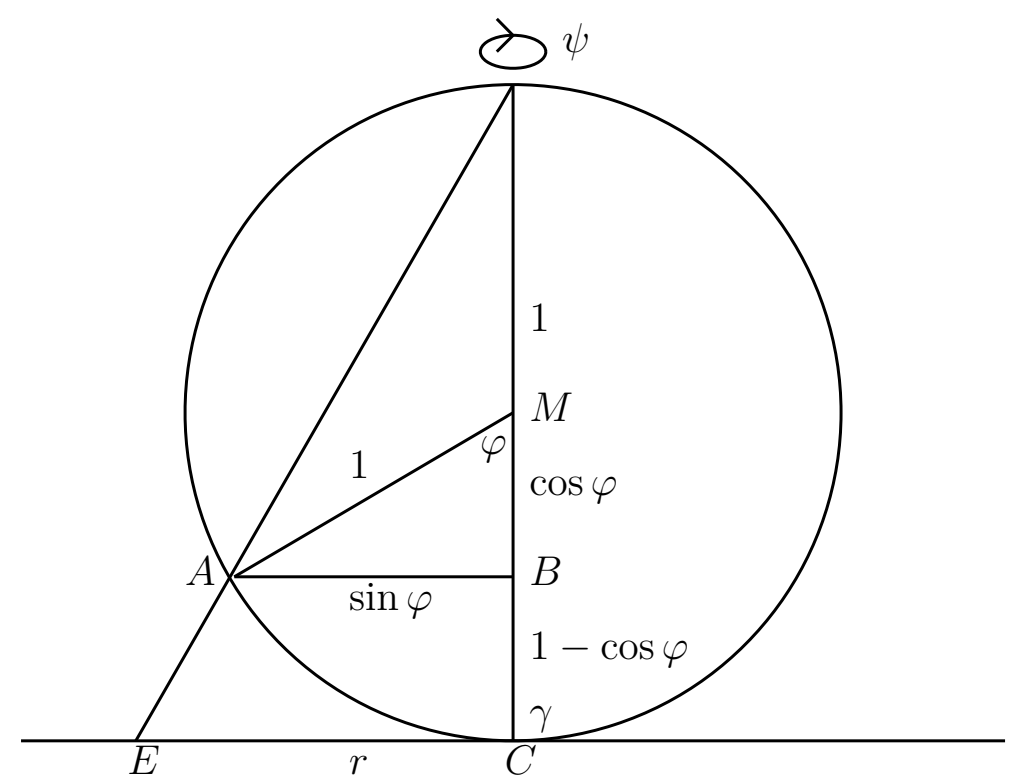

Figure 6: Sketch of the inversion wrt. the sphere $\Omega$

and hence

$$
w(x, y)=\frac{4}{r\left(4+r^{2}\right)} \sin \varphi=\frac{4}{r\left(4+r^{2}\right)} \frac{4 r}{4+r^{2}}=\left(\frac{4}{4+r^{2}}\right)^{2},
$$

where some transformations of (16) lead to an expression of $\sin \varphi$ in terms of $r$. Now it holds

$$
\begin{aligned}
\int_{\Gamma} f(\xi) g(\xi) d \omega(\xi) & =\int_{(x, y) \in \Upsilon_{\gamma}(\Gamma)}(f g)\left(\Upsilon_{\gamma}((x, y))\right) w(x, y) d x d y \\
& =\int_{(x, y) \in \Upsilon_{\gamma}(\Gamma)} \Theta_{\gamma}[f]((x, y)) \Theta_{\gamma}[g]((x, y)) W(x, y) d x d y
\end{aligned}
$$

where due to $(9)$

$$
W(x, y)=w(x, y)\left(\frac{\sqrt{r^{2}+4}}{2}\right)^{2}=\left(\frac{4}{r^{2}+4}\right)^{2} \frac{r^{2}+4}{4}=\frac{4}{r^{2}+4}
$$

which is exactly the last assertion.

Proof of Lemma 2.8:

Trivial consequence of

$$
\begin{aligned}
\frac{\partial}{\partial \varphi} f((\varphi, \psi)) & =\frac{\partial}{\partial \varphi} \Theta_{\gamma}\left[\Theta_{\gamma}[f]\right]((\varphi, \psi)) \\
& =\frac{\partial}{\partial \varphi}\left(\frac{1}{\sqrt{2} \sqrt{1+\cos \varphi}} \Theta_{\gamma}[f]\left(\Upsilon_{\gamma}((\varphi, \psi))\right)\right)
\end{aligned}
$$

The same holds for the derivative in $\psi$ direction. 


\section{References}

[1] J.-P. Antoine, L. Demanet, L. Jacques, and P. Vandergheynst, Wavelets on the sphere : Implementation and approximations, Appl. Comput. Harmon. Anal. 13 (2002), no. 3, 177-200.

[2] J.-P. Antoine and P. Vandergheynst, Wavelets on the n-sphere and other manifolds, J. Math. Phys. 39 (1998), 3987-4008.

[3] J.-P. Antoine and P. Vandergheynst, Wavelets on the 2-sphere: A group-theoretical approach, Appl. Comput. Harmon. Anal. 7 (1999), 262-291.

[4] S. Axler, P. Bourdon, and W. Ramey, Harmonic function theory, second ed., Graduate Texts in Mathematics, vol. 137, Springer-Verlag, New York, 2001.

[5] M. de Berg, M. van Kreveld, M. Overmars, and O. Schwarzkopf, Computational geometry, second ed., Springer-Verlag, New York, 2000.

[6] G. Bernardes, S. Bernstein, P. Cerejeiras, and U. Kähler, Wavelets invariant under finite reflection groups, Math. Methods Appl. Sci. 33 (2009), no. 4, 473-484.

[7] H. S. M. Coxeter, Introduction to geometry, Wiley Classics Library, John Wiley \& Sons Inc., New York, 1989, Reprint of the 1969 edition.

[8] S. Dahlke, W. Dahmen, E. Schmitt, and I. Weinreich, Multiresolution analysis and wavelets on $s^{2}$ and $s^{3}$, Numer. Funct. Anal. Optim. 16 (1995), 19-41.

[9] S. Dahlke and P. Maass, Continuous wavelet transforms with application to analyzing functions on spheres, J. Fourier Anal. Appl. 2 (1996), 379-396.

[10] I. Daubechies, Ten Lectures on Wavelets, CBMS-NSF Regional Conference Series in Applied Mathematics. 61. Philadelphia, PA: SIAM, Society for Industrial and Applied Mathematics. xix, 357 p. , 1992.

[11] W. Freeden, T. Gervens, and M. Schreiner, Constructive approximation on the sphere (with applications to geomathematics), Numerical Mathematics and Scientific Computation, Oxford University Press, Oxford, New York, 1998.

[12] W. Freeden and M. Schreiner, Biorthogonal locally supported wavelets on the sphere based on zonal kernel functions, J. Fourier Anal. Appl. 13 (2007), 693-709.

[13] J. Göttelmann, Locally supported wavelets on manifolds with applications to the $2 d$ sphere, Appl. Comput. Harmon. Anal. 7 (1999), 1-33.

[14] H. Harbrecht and R. Schneider, Wavelet galerkin schemes for boundary integral equations - implementation and quadrature, SIAM J. Sci. Comput. 27 (2006), no. 4, $1347-1370$.

[15] M. Holschneider, Continuous wavelet transforms on the sphere, J. Math. Phys. 37 (1996), 4156-4165.

[16] M. Jansen and P. Oonincx, Second generation wavelets and applications, Springer, Berlin, 2005. 
[17] A. Kunoth and J. Sahner, Wavelets on manifolds: An optimized construction, Math. Comp. 75 (2006), 1319-1349.

[18] F. G. Lemoine, S. C. Kenyon, J. K. Factor, R. G. Trimmer, N. K. Pavlis, D. S. Chinn, C. M. Cox, S. M. Klosko, S. B. Luthcke, M. H. Torrence, Y. M. Wang, R. G. Williamson, E. C. Pavlis, R. H. Rapp, and T. R. Olson, The Development of the Joint NASA GSFC and NIMA Geopotential Model EGM96, NASA/TP-1998206861, NASA Goddard Space Flight Center, Greenbelt, Maryland, USA, 1998.

[19] S. Mallat, A wavelet tour of signal processing, Academic Press Inc., San Diego, CA, 1998.

[20] F.J. Narcowitch and J. D. Ward, Nonstationary wavelets on the m-sphere for scattered data, Appl. Comput. Harmon. Anal. 3 (1996), 1324-1336.

[21] D. Potts, G. Steidl, and M. Tasche, Fast Fourier transforms for nonequispaced data: a tutorial, Modern sampling theory, Appl. Numer. Harmon. Anal., Birkhäuser Boston, Boston, MA, 2001, pp. 247-270.

[22] R. Rummel, G. Balmino, J. Johannessen, P. Visser, and P. Woodworth, Dedicated gravity field missions - principles and aims, J. Geodyn. 33 (2002), 3 - 20.

[23] R. Rummel, C. Reigber, and K. Ilk, The use of satellite-to-satellite tracking for gravity parameter recovery, ESA Workshop on Space oceanography, Navigation and Geodynamics (SONG), ESA-SP-137, 1978, pp. 151-161.

[24] F.J. Simons, Open source software for spherical harmonics in matlab, http://geoweb.princeton.edu/people/simons/sof tware.html.

[25] F.J. Simons and F. Dahlen, Spherical slepian functions and the polar gap in geodesy, Geophys. J. Int. 166 (2006), 1039-1061.

[26] A. Tagliasacchi, Kd-tree for matlab, http://www . mathworks. com/matlabcentral/fileexchange/21512.

[27] Y. Wiaux, L. Jacques, and P. Vandergheynst, Correspondence principle between spherical and euclidean wavelets, Astrophys. J. 632 (2005), 15-28.

[28] Y. Wiaux, J. D. McEwen, P. Vandergheynst, and O. Blanc, Exact reconstruction with directional wavelets on the sphere, Mon. Not. R. Astron. Soc. 388 (2008), $770-788$.

[29] R. S. Womersley and I. H. Sloan, How good can polynomial interpolation on the sphere be?, Adv. Comput. Math. 14 (2001), 195-226. 